\title{
Density holes in the upstream solar wind
}

\author{
G. K. Parks ${ }^{1}$, E. Lee ${ }^{1}$, N. Lin ${ }^{1}$, F. Mozer ${ }^{1}$, M. Wilber ${ }^{1}$, E. Lucek ${ }^{2}$, \\ I. Dandouras ${ }^{3}$, H. Rème ${ }^{3}$, J. B. $\mathrm{Cao}^{4}$, P. Canu ${ }^{5}$, N. Cornilleau-Wehrlin ${ }^{5}$, \\ P. Décréau ${ }^{6}$, M. L. Goldstein ${ }^{7}$ and Philippe Escoubet ${ }^{8}$ \\ ${ }^{1}$ Space Sciences Laboratory, University of California, Berkeley, CA, USA \\ ${ }^{2}$ Blackett Laboratory, Imperial College, London, UK \\ ${ }^{3}$ Centre d'Etude Spatiale des Rayonnement, Paul Sabatier University, Toulouse, France \\ ${ }^{4}$ Key Laboratory for Space Weather, CSSAR, CAS, Beijing, China \\ ${ }^{5}$ CETP, Velizy, France \\ ${ }^{6}$ LPCE and University of Orleans, Orleans, France \\ ${ }^{7}$ NASA Goddard Space Flight Center, Greenbelt, MD, USA \\ ${ }^{8}$ European Space Agency, Noordwijk, The Netherlands
}

\begin{abstract}
Larmor size transient structures with depletions as large as $99 \%$ of ambient solar wind density levels occur commonly upstream of Earth's collisionless bow shock. These "density holes" have a mean duration of $\sim 17.9 \pm 10.4 \mathrm{~s}$ but holes as short as $4 \mathrm{~s}$ have been observed. The average fractional density depletion $(\delta \mathrm{n} / \mathrm{n})$ inside the holes is $\sim 0.68 \pm 0.14$. The density of the upstream edge moving in the sunward direction can be enhanced by five or more times the solar wind density. Particle distributions show the steepened edge can behave like a shock, and measured local field geometries and Mach number support this view. Similarly shaped magnetic holes accompany the density holes indicating strong coupling between fields and particles. The density holes are only observed with upstream particles, suggesting that back-streaming particles interacting with the solar wind are important.
\end{abstract}

Keywords: Density hole

PACS: 95.30.Qd, 96.50 Tf, 96.60 Vg

\section{INTRODUCTION}

This article summarizes the characteristics of Larmor radius scale transient structures recently discovered in the upstream region of Earth's bow shock [1]. These structures, called density holes, are seen very frequently in the upstream region and may be important for understanding how the collisionless bow shock forms.

Figure 1 shows typical data from the ion and magnetic field experiments on the European Cluster-1 (top) and Chinese Double Star (DS) satellites in the vicinity of the bow shock. The magnetosheath in the energy flux spectrogram is a broad energy band while the solar wind is indicated by the narrow red band, at $\sim 2 \mathrm{keV} / \mathrm{q}$. Cluster 1 (DS) crossed the bow shock at $\sim 0829$ UT ( $\sim 0922$ UT) but moved in and out and the shock and magnetosheath plasma was detected several times. The weaker fluxes above the few keV/q solar wind are the backstreaming particles. The solar wind speed on this day was $\sim 600 \mathrm{~km} / \mathrm{s}$ in the $-V_{\mathrm{x}}$ direction with a density $\mathrm{n} \sim 2.5 \mathrm{~cm}^{-3}$.

The sharp dips in $n$ that go below the average solar wind density $\left(\mathrm{n}_{\mathrm{sw}}\right)$ are density holes. The holes are followed by overshoots above $n_{s w}$ ( 0855 and 0901 UT). Examination of data from many crossings indicates that the holes appear in bunches 
close to the bow shock, but more in isolation further away. Density holes have been seen all the way to Cluster apogees $\left(\sim 19 \mathrm{R}_{\mathrm{E}}\right)$. The bulk velocities accompanying these dips behave as they do near the bow shock: $V_{x}$ decreases almost to zero, and $V_{y}$ and $V_{z}$ deviate substantially. $T$ increases by an order of magnitude.
$\mathrm{CIS}-\mathrm{HIA}$
RUMBA (SC 1)
$02 / \mathrm{Mar} / 2005$
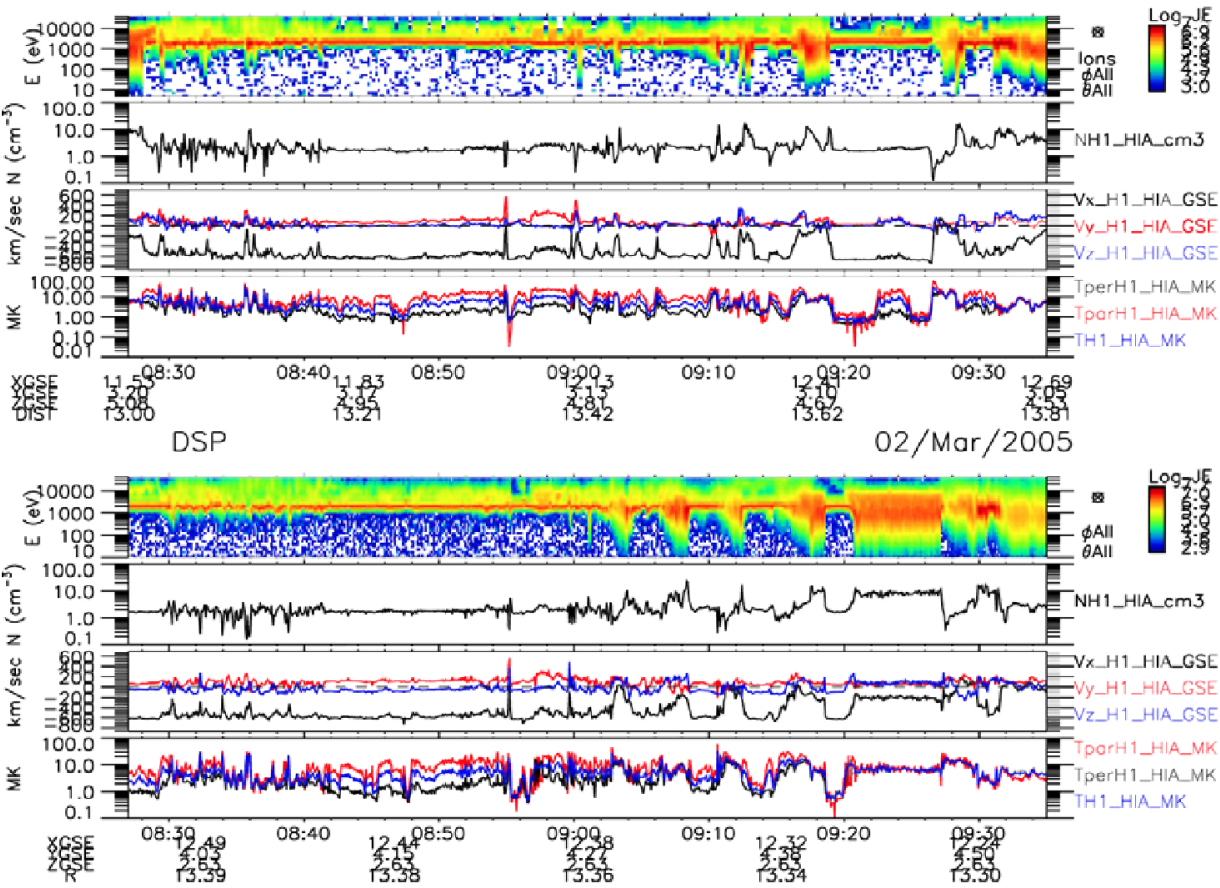

Figure 1: Cluster 1 and the Double Star observed nearly identical density hole structures, for example, at 0856 UT. Cluster 1 was outbound and Double Star inbound. The locations of the spacecraft are shown at the bottom (GSE coordinates). The panels from top to bottom show energy flux, density, velocity and temperature.

The right panel of Fig. 2 shows spin resolution (4s) data of a density hole detected at 0855 UT on March 2, 2005 by Cluster 1 (Double Star also detected this hole; not shown). For this hole, $\mathrm{n}$ at both edges increased from the solar wind value $\sim 2$ to $\sim 3.5$ $\mathrm{cm}^{-3}$, while inside the hole, it decreased to $\sim 0.45 \mathrm{~cm}^{-1}$. The bulk T rose from $\sim 3 \times 10^{5}$ ${ }^{\circ} \mathrm{K}$ to $\sim 2 \times 10^{7}{ }^{\circ} \mathrm{K}$ and $\mathrm{V}_{\mathrm{x}}$ decreased from about $-600 \mathrm{~km} \mathrm{~s}^{-1}$ to $\sim 0 \mathrm{~km} / \mathrm{s}$ while $\mathrm{V}_{\mathrm{y}}$ increased from $\sim 100 \mathrm{~km} \mathrm{~s}^{-1}$ to $\sim 600 \mathrm{~km} \mathrm{~s}^{-1}$. The solar wind parameters were, $\beta=$ $8 \pi \mathrm{nkT} / \mathrm{B}^{2} \sim 1.6, \mathrm{~T}_{\mathrm{e}} / \mathrm{T}_{\mathrm{i}} \sim 2, \mathrm{~V}_{\mathrm{A}}=\mathrm{B} /\left(4 \pi \mathrm{nm}_{\mathrm{i}}\right)^{1 / 2} \sim 56 \mathrm{~km} \mathrm{~s}^{-1}$ and $\mathrm{C}_{\mathrm{s}}=\left(\mathrm{kT}_{\mathrm{e}} / \mathrm{m}_{\mathrm{i}}\right)^{1 / 2} \sim 90 \mathrm{~km} \mathrm{~s}^{-1}$.

Ion density holes are accompanied by magnetic holes of nearly the same shape. The left panel of Figure 2 shows a Lagrangian view of this density hole. A timing analysis from the four Cluster spacecraft assuming a planar geometry yielded a normal $(-0.65$, $0.75,0.065$ ) for the downstream edge at $0855 \mathrm{UT}$ and a speed of $\sim 530 \mathrm{~km} \mathrm{~s}^{-1}$ along the 
normal. The normal of the upstream edge was $(-0.80,0.59,0.11)$ with an antisunward speed $\sim 320 \mathrm{~km} \mathrm{~s}^{-1}$, indicating a significant relative motion. Detection times for the edges by Double Star downstream indicated that the speed of the edges could have changed. The resulting downstream edge acceleration was estimated to be $\sim 56 \mathrm{~km} \mathrm{~s}^{-2}$, and the sunward edge deceleration $-23 \mathrm{~km} \mathrm{~s}^{-2}$. This implies the hole was growing at an increasing rate (Timing errors are $\sim 10 \%$. In the case of acceleration, the errors are larger).

The size of the downstream edge at Cluster was deduced to be $\sim 1300 \mathrm{~km}$, the upstream edge $\sim 2300 \mathrm{~km}$, and the hole $\sim 3700 \mathrm{~km}$ (Including acceleration would make them larger). The gyroradius of $4 \mathrm{keV}$ proton in $\mathrm{B}=4 \mathrm{nT}$ is $\sim 1100 \mathrm{~km}$. The growth time of the hole, obtained by dividing the width of the hole by the relative velocity of the two edges yields $\sim 10$ s (including acceleration will reduce this value). The $|\mathbf{B}|$ variations were in phase with the density but the $\mathbf{B}$ intensity at the downstream edge was $\sim 20 \%$ larger than the exterior field $(\sim 4.5$ to $\sim 5.5 \mathrm{nT})$, while at the upstream edge it nearly doubled, $\sim 4 \mathrm{nT}$ to $\sim 7 \mathrm{nT}$. Inside the hole, B decreased to $\sim 0.5 \mathrm{nT}$.
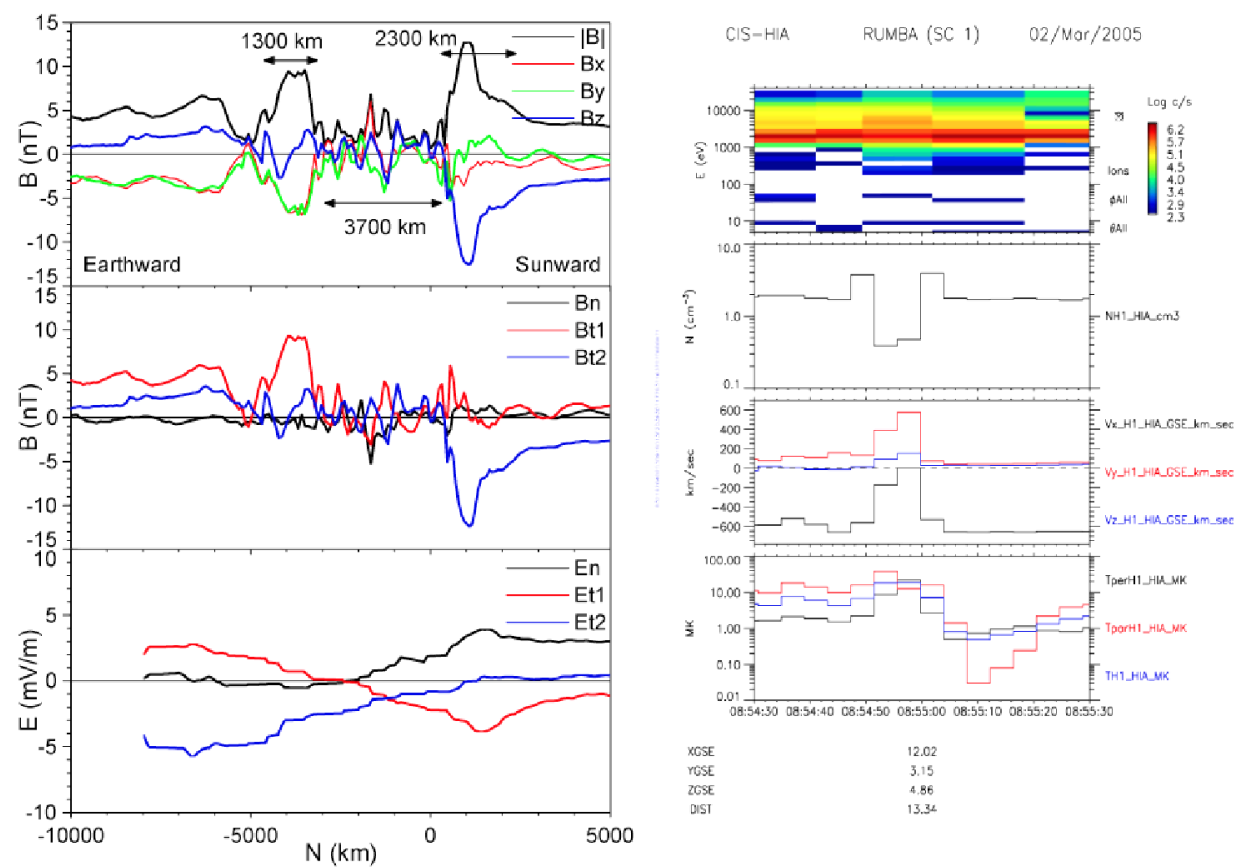

Figure 2: A Langrangian view (left) of a density hole (right) observed by Cluster 1. The E-field data were averaged and high frequency variations were removed. The density holes are accompanied by nearly identically shaped magnetic holes with reduced intensity in the hole and steepened $B$ at the edge. The scale of $\mathbf{B}$ is linear while for $\mathbf{n}$ it is logarithmic. Temperature $\mathrm{T}$ inside the hole is about 100 times the solar wind $T$. The GSE coordinates of Cluster 1 are $(12.0,3.2,4.9) \mathrm{R}_{\mathrm{E}}$.

The $\mathbf{B}$ at both edges was compressed and the normal component $B_{n}$ nearly vanished throughout. $\mathbf{E}$ included both tangential $E_{t}$, and normal $E_{n}$ with $E_{t}$ perpendicular to $B_{t}$. 
Here $E_{t}=E_{t 1}+E_{t 2}$. Measurements of $\mathbf{B}$ inside the hole indicated presence of small and fluctuating field (Small fluctuations are also seen in $\mathbf{E}$ but data have been smoothed). This density hole is a published SLAMS event and the behavior of $\mathbf{E}$ and $\mathbf{B}$ has been studied [2-4]. SLAMS have been studied in detail using magnetic field data but not the ions associated with them.

Power spectra obtained from two wave experiments $[5,6]$ for the density hole discussed above are complex and not all understood (Figure 3). Wave power for $\delta \mathbf{E}$ and $\delta \mathbf{B}$ was considerably enhanced inside and at the edges of the density hole. For $\delta \mathbf{B}$, power inside the hole was enhanced from a few $\mathrm{Hz}$ to close to the ion cyclotron frequency $(\sim 0.01 \mathrm{~Hz}-0.1 \mathrm{~Hz})$ while enhancement for $\delta \mathbf{E}$ was observed at a few $\mathrm{kHz}$. These waves are in the same frequency range as the Doppler shifted ion acoustic waves frequently observed in the solar wind. We also see enhanced power for $\delta \mathbf{E}$ above the electron cyclotron frequency inside the hole. These waves are not yet identified since electrostatic waves above the electron cyclotron frequency have not been seen previously in the solar wind (to our knowledge).

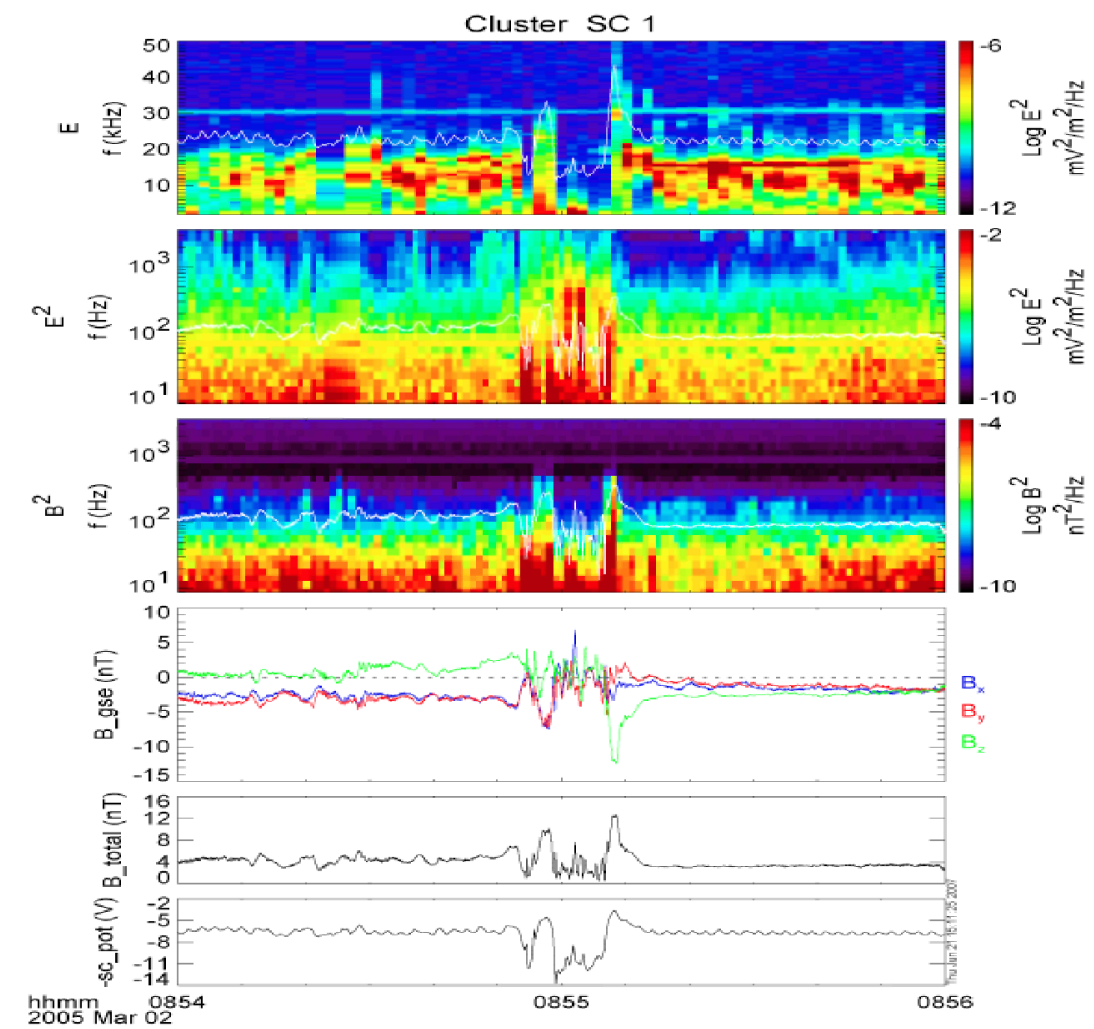

FIGURE 3. Top three panels show power spectra of $\delta \mathrm{E}$ from $\sim 2-50 \mathrm{kHz}$. (white line is estimated plasma frequency) and sum of $\delta \mathrm{E}$ in spin plane from $8 \mathrm{~Hz}-4 \mathrm{kHz}\left(\mathrm{mV}^{2} / \mathrm{m}^{2} / \mathrm{Hz}\right)$ and sum of $\delta \mathrm{B}$ $\left(\mathrm{nT}^{2} / \mathrm{Hz}\right)$. White line is calculated electron cyclotron frequency. The bottom three panels show magnetic field components and magnitude and the spacecraft potential that is a proxy for electron density. 
The waves enhanced at the edges for $\delta \mathbf{B}$ and $\delta \mathbf{E}$ cover the frequency range a few $\mathrm{Hz}$ to several hundred $\mathrm{Hz}$ (up to electron cyclotron frequency) and the waves include whistler mode waves. The burst of broadband emissions observed up to the electron plasma frequency are probably associated with electrostatic solitary structures traveling along $\mathbf{B}$.

An ion distribution associated with the density hole at 0855 UT (March 2, 2005) is shown in Figure 4 (left panel). This velocity space plot is a cut normal to B through the main solar wind beam (centered) and shows the non-gyrotropic character of the plasma (B points out of the page.) This 3-spin (12 s) integration overlaps the upstream edge of the structure and is time-aliased, but still reveals an arc of ions in the upper left quadrant that appears to be a gyro-reflected component similar to those seen at the foot of collisionless shocks. Using the upstream sunward edge normal direction and the speed from timing analysis, we obtained a normal incidence Alfvén Mach number $\mathrm{M}_{\mathrm{A}}=3.3$. The upstream exterior $\mathrm{B}$ was about $(-1,-1,-3) \mathrm{nT}$, which was $>80^{\circ}$ from the normal direction, indicating the solar wind flow into this structure was supercritical with a strongly perpendicular geometry. We thus have good reason to expect that a shock-like boundary might form, and the presence of reflected ions suggests an associated cross-boundary E-field as well (Figure 2)
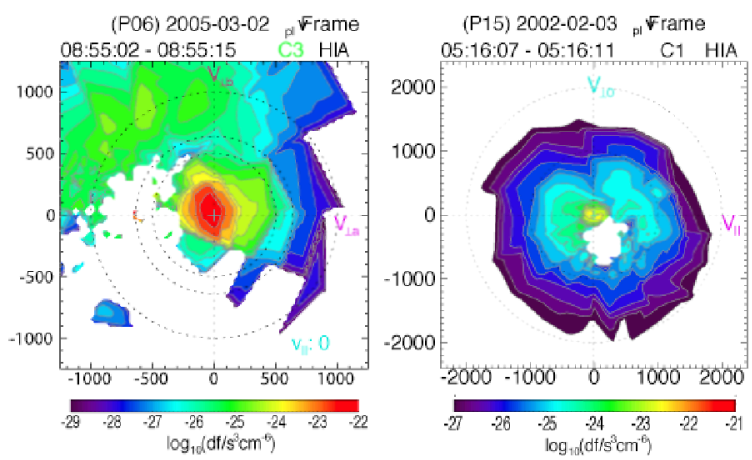

Figure 4: Ion phase space distributions of density holes. Left panel: a B-perpendicular cut through the main solar wind beam in the upstream edge of the 0855 UT, 2 March 2005 event. The field points out of the page and the horizontal axis points nearly anti-sunward. Most particles have relatively modest energies, and the velocity scale includes only $+/-1250 \mathrm{~km} \mathrm{~s}^{-1}$ to highlight their behavior. Right panel: a $\mathbf{V}_{\mathrm{sw}} \times \mathbf{B}$ slice through a distribution obtained within the interior of the 0516 UT, 3 February 2002 density hole. B points to the right (Reprinted from [1]).

A feature not apparent in the left panel of Figure 4 is revealed in a distribution (right panel) obtained interior to a density hole observed at $\sim 0516$ UT on 3 February 2002, identified as a SLAMS (Short Large-Amplitude Magnetic Structures) event [7]. In this plot, the slice is through a plane defined by $\mathbf{V}_{\mathrm{sw}} \times \mathbf{B}$ with $\mathbf{B}$ pointing to the right, and again the main solar wind beam is centered. Comparisons with upstream distributions obtained a few spins later show that the main solar wind beam component is reduced in phase space density by more than an order of magnitude, 
consistent with the large reduction in overall density $\mathrm{n}$. Also present is a significant suprathermal population that appears much like an upstream intermediate distribution. The temperature in density holes rose to $>10^{7} \mathrm{~K}$, and can be accounted for by the presence of this energetic component and the reduction of the colder solar wind population. Note that while the solar wind beam is observed to slow down and change direction within and at the edges of the density hole, the measured beam velocity differs somewhat from the computed moments, indicating relative motion of the energetic component. The latter ions may originate in backstreaming particles observed exterior to the structure, retaining some of their initial field-aligned momentum.

\section{DISCUSSION}

Several characteristics of density holes are similar to those of HFAs (Hot Flow Anomalies), HDCs (Hot Diamagnetic Cavities) and SLAMS (TABLE 1). They all have significant bulk flow deflections, are filled with heated plasmas, have enhanced edge densities, and one or both edges have compressed magnetic field. Density holes durations are similar to SLAMS but much shorter than HFAs and HDCs, and correspondingly smaller dimensions. The order of magnitude density depletions have not been reported previously for HFAs. HDCs or SLAMS. Density holes are common while HFAs and HDCs occur rarely. Only eight HFA events had been reported in ISEE observations spanning more than two years, and the combined number including HDCs observed by ISEE, AMPTE-IRM and AMPTE-UKS was about 30 [8-10].

Table 1: Upstream Transient Structures

\begin{tabular}{|l|l|l|l|l|}
\hline Property & $\mathrm{DH}$ & HFA/HDC & SLAMS & FC \\
\hline Duration & $\sim 18 \mathrm{~s}$ & $\sim$ few minutes & $\sim 10-20 \mathrm{~s}$ & $>$ few minutes \\
\hline Scale length & $\mathrm{R}_{\perp}$ & $\sim \mathrm{R}_{\mathrm{E}}$ & $\sim \mathrm{R}_{\mathrm{E}}$ & $>\mathrm{R}_{\mathrm{E}}$ \\
\hline$\delta \mathrm{n} / \mathrm{n}$ & $\sim 0.7$ & $<0.2$ & No report & $<0.2$ \\
\hline Bulk V & $\mathrm{Vx} \rightarrow 0$ & $\mathrm{Vx} \rightarrow-100$ & No report & $\sim \mathrm{Vsw}$ \\
\hline T (hole) & $\sim 10^{7}$ & $\sim 10^{6}$ & No report & $>103$ \\
\hline Overshoot & Yes & Yes & Yes & Yes \\
\hline Shock-like & Yes & Yes & Yes & No \\
\hline Occurrence & Frequent & Rare & Frequent & Rare \\
\hline E-field & Yes & No report & Yes & No report \\
\hline Upstream ions & Yes & Yes & No report & No report \\
\hline Electron hole & Yes & No report & No report & No report \\
\hline Waves & Yes & No report & Yes & No report \\
\hline Currents & Yes & No report & Inferred & No report \\
\hline
\end{tabular}


We have shown one example of SLAMS event that has a density hole with "heated" back-streaming plasmas associated with it. In addition, the density holes near the shock tend to have elevated edges that account for more particles than those depleted within the hole. The heating plus the net gain in particles is consistent with what is observed across the shock transition, and reflects as expected net density increases within structures moving relative to the solar wind flow.

Density holes are observed only when back-streaming energetic particles are present although the latter can exist without the former. These intervals usually have B perturbations. However, the details involved in creating the density holes are not known. Density holes have been observed for solar wind velocities $<400$ to $>800 \mathrm{~km}$ $\mathrm{s}^{-1}, \mathrm{n} \sim 1-10 / \mathrm{cc}$, and $\mathrm{T} \sim 10^{5}-10^{6}{ }^{\circ} \mathrm{K}$. While $\mathbf{B}$ and $\mathrm{n}$ are correlated, deep density holes with large edge enhancements can have shallow magnetic holes and weak B overshoots, while shallow density holes with weak edge enhancements can have deep magnetic holes and large overshoots. We will continue to study the electromagnetic and electrostatic wave data and particle observations in order to establish the roles of wave particle interactions. Improved statistics on upstream plasma and beam parameters (density, speed, $T$, Mach number, plasma $\beta$ and shock geometry) may reveal factors controlling their origin and development.

\section{ACKNOWLEDGEMENT}

The research at UC Berkeley is performed under the auspices of a NASA Grant No. NNG04GF23G. Cluster is a joint project of ESA and NASA and Double Star a joint project of ESA and the Chinese Space Agency.

\section{REFERENCES}

1. G. K. Parks, E. Lee, N. Lin, et al., Phys. of Plasma. 13, 050701-1 (2006).

2. E. A. Lucek et al., Ann. Geophys. 20, 1699 (2002).

3. E. A. Lucek et al., Ann. Geophys. 22, 1 (2004).

4. E. Lucek, T. S. Horbury and Balogh et al., J. Geophys. Res., 109, A06207 (2004).

5. N. Cornilleau-Wehrlin et al., Ann. Geophys.,21, 437 (2003).

6. P. Decreau et al., Ann. Geophys., 19, 1241 (2001).

7. R. Behlke et al., Geophys. Res. Lett., 30, No. 4, 1177 (2003).

8. M. F. Thomsen, J. T. Gosling, et al., J. Geophys. Res., 91, 2961 (1986).

9. S. J. Schwartz et al., J. Geophys. Res., 97, 4209 (1992).

10. S. J. Schwartz et al., J. Geophys. Res., 105, 12639 (2000) 